\title{
Analysis of the results of the first implementation of the Korea Manuscript Editors Certification
}

\author{
Hyun Jung Yi' ${ }^{1}$ Jae Hwa Chang' ${ }^{2}$, Yoon Joo Seo ${ }^{3}$ \\ ${ }^{1}$ Medical Library, Hangyang University Guri Hospital, Guri; ${ }^{2}$ Infolumi, Seongnam; ${ }^{3}$ MEDrang Inc., Seoul, Korea
}

\section{Abstract}

In the field of international scholarly journal publishing, manuscript editing has been established as an essential component of the publication process. As the necessity of this process has increased, the Korean Council of Science Editors has consistently provided education for training professional manuscript editors, and has worked to implement a manuscript editor certification system. Starting in 2014, the Korean Council of Science Editors thoroughly conducted background research and advanced analysis in preparation for such a system. Subsequently, a committee of experts was formed to develop and simulate an examination for this certification. This process culminated in the first manuscript editor certification examination, which was held in November 2016 and resulted in 40 initial Korea Manuscript Editors Certification holders. Examinations for the Korea Manuscript Editors Certification are scheduled to be held annually. The establishment of this certification system will contribute to strengthening individual capacities and further developing science journal publication in Korea by expanding the field of manuscript editing. Ultimately, this system will contribute to the promotion of Korean scientific journals to the level of prominent international journals.

Keywords

Academic publishing; Certification; Manuscript editor

Received: February 1,2017 Accepted: February 6, 2017

Correspondence to Yoon Joo Seo milbory@medrang.co.kr

ORCID

Hyun Jung Yi

http://orcid.org/0000-0003-0663-4373 Jae Hwa Chang

http://orcid.org/0000-0002-2922-9197

Yoon Joo Seo

http://orcid.org/0000-0002-0202-8352

\section{Introduction}

Manuscript editors (MEs; also known as copy editors, technical editors, or managing editors) check a manuscript to ensure that it adheres to the proper format for a journal's style and play an essential role in the international academic environment.

ME certificate or certification systems have already been widely established internationally. Several representative institutions or editors' associations, including the Board of Editors in the Life Sciences (BELS), American Medical Writers Association (AMWA), and Council of Sci- 
ence Editors, provide active education for training professional MEs and issue certificates to MEs who meet appropriate criteria.

As Korean science journals quickly rose to an international status, the style and format of journals became important criteria in their evaluation. Therefore, interest in manuscript editing and the necessity of professional MEs have continually increased.

However, due to the lack of standards regarding the roles and qualifications of MEs, journal editorial boards have articulated an increasing demand for clear standards and proper education. Hence, the Korean Council of Science Editors (KCSE) implemented an ME certification system.

\section{Utilization of the Korea Manuscript Editors Certification}

The Korea Manuscript Editors Certification (KMEC) is the first private ME certification system adopted by the KCSE, and aims to train and certify MEs in the field of science and technology in Korea. This certification is issued to those who have a qualified educational background, a certain amount of work experience, have received sufficient educational credits from the KCSE, and then pass the annual test.

This will provide an effective learning opportunity and motivation to MEs and journal publishing personnel, along with the public acknowledgement of an individual's expertise. Meanwhile, this program is expected to play an important role not only for individual MEs, but also for journal publishers in the field of science. The KCSE guarantees that MEs with this certificate have core competencies in manuscript editing, so journal publishers will be able to refer to this certificate when they want to hire a skilled ME.

Those who acquire the KMEC will be updated regarding worldwide trends in manuscript editing and professional education programs. Additionally, it is expected that those with the KMEC will be able to further develop their skills by actively exchanging useful information within the ME community [1].

\section{Exam Development and Implementation Process}

The KCSE thoroughly conducted background research and advanced analysis in preparation for the KMEC system. Subsequently, a committee of experts was formed to develop and simulate the examination. This article summarizes the implementation of the examination, and analyzes its composition and results.

The KCSE began to discuss the necessity of a certification system in 2014, and conducted background research. It checked whether a domestic certification regarding MEs ex- isted, and reviewed foreign $\mathrm{ME}$ certifications, including the BELS, AMWA, and Council of Science Editors programs. Moreover, other domestic certifications, such as the medical librarian certification system, were examined as well.

In 2015, a survey regarding the demand for and validity of an ME certification was conducted among Korean science journal editors [2]. A fundamental study based on the survey confirmed that the certification system was valid enough for an ME certification to be implemented, so the KCSE decided to introduce an ME certification system suitable for the current state of scientific journals in Korea. Accordingly, a fullscale effort to develop an examination began in 2016.

After comprehensive discussion, an examination committee comprising 1 chairman, 2 counsels, and 11 examiners was established in July 2016. This committee was founded by members with all the following qualifications: 1) at least 3 years of experience in professional manuscript editing or journal publication; 2) a certification issued by BELS or AMWA, or completion of a formal ME education from a prominent university; and 3) a sufficient amount of educational credits received through the KCSE.

In September 2016, all examiners wrote 30 to 50 questions each, and 100 sample questions were selected after peer review and revision at the committee workshop. In October 2016, a simulation test using the sample questions was conducted on 4 current MEs with more than 3 years of experience. The level of difficulty and examination timing were subsequently adjusted. Eventually, the final version of the examination was completed with the assistance of KCSE members and other experts.

Individuals interested in or engaged in scientific journal publication were informed about the purpose and format of the examination, as well as the relevant qualifications, through the KCSE website and e-mail by August 2016. Those with a bachelor's degree or higher in any major, more than a year of manuscript editing experience, and more than 20 credits of relevant education within the past 3 years were qualified to take the examination.

\section{Format and Content of the Examination}

The first KMEC examination was held on November 19, 2016 in Seoul. By 8:50 a.m., 37 candidates had completed their registration, and the test paper was distributed after 30 minutes of instruction. The candidates were given 100 questions and the non-open-book test lasted for 2 and a half hours in the presence of 3 KCSE members (Fig. 1).

All the questions were in a 4-option multiple choice format and covered a wide range of content, including the following topics: the role of MEs, instructions for authors, copyediting 
skills according to a the given style guide, bibliographic references and citations, elements of scientific publication, tables and figures, statistics, publication ethics and copyright issues, databases and impact factor, and punctuation.

The content was divided into 12 different domains, and the

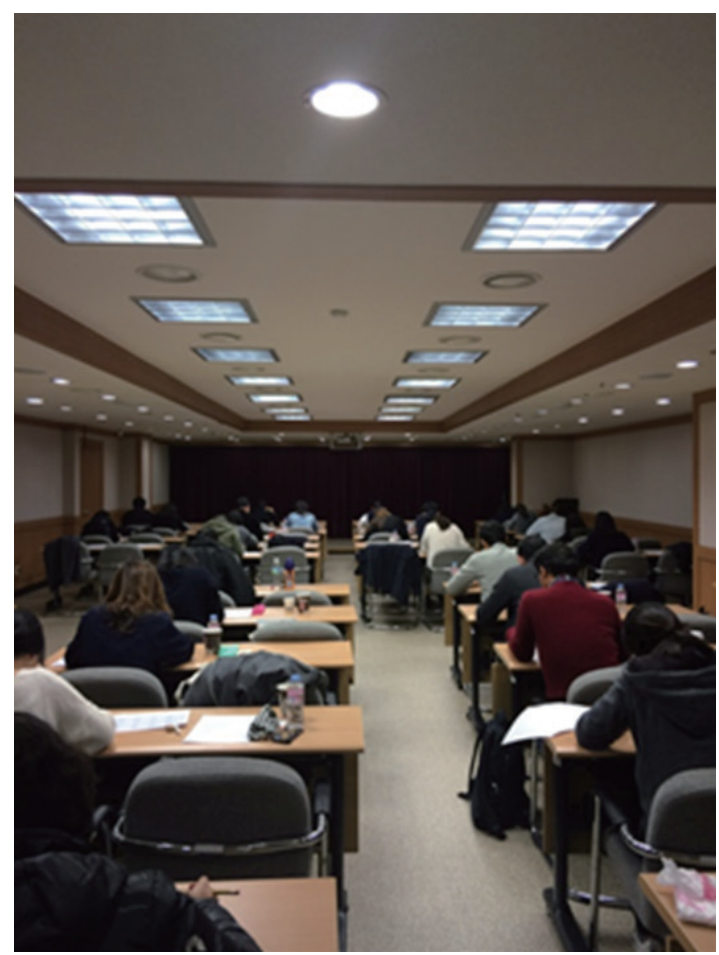

Fig. 1. First Korea Manuscript Editor Certification Examination. The candidates were concentrating on the exam.

Table 1. Domains of the questions

\begin{tabular}{lc}
\hline Domain & No. of questions \\
\hline Role of the manuscript editor & 3 \\
General corrections & 9 \\
Units and abbreviations & 12 \\
References & 8 \\
Statistics & 4 \\
Tables and figures & 10 \\
Understanding the elements of a research article & 8 \\
Publication ethics and copyright & 8 \\
Technical factors related to publishing & 8 \\
Databases and citation indices & 10 \\
Korean grammar & 5 \\
English grammar and punctuation & 15 \\
Total & 100 \\
\hline
\end{tabular}

number of questions in each domain varied (Table 1). Units and abbreviations and English grammar and punctuation were the domains with the most questions because they are directly related to essential work skills in manuscript editing.

\section{Results}

The KMEC is fundamentally an absolute evaluation system requiring candidates to score at least 70 correct answers out of 100. However, the candidates were informed that the passing grade may change according to the difficulty of the examination. Among the 50 applicants who received the qualification, 11 were certified without an examination, and 37 of the remaining 39 (95\%) took the examination. Twenty-nine of those 37 candidates passed the examination, indicating a passing rate of $78 \%$.

Eight questions exhibited a 100\% correct answer rate, 35 had a correct response rate of over $90 \%$, and 51 had a correct answer rate of over $80 \%$ (Table 2). However, one question had a correct response rate of only $16.2 \%$ ( 6 of 37). This question was included in the English grammar and punctuation domain, and asked which instructions of the journal a given paragraph belonged to.

Eighteen questions had a correct response rate of under $50 \%$. Questions asking for the correct explanation of a Latin abbreviation, the selection of a database that provides full-text articles, and identification of a misused punctuation mark each showed a $27 \%$ correct answer rate. The results for each domain are presented in Table 3. The units and abbreviations domain and the English grammar and punctuation domain, which contained the largest number of questions, also had the largest number of questions with low correct answer rates.

Table 2. Number of questions with various correct answer rates

\begin{tabular}{lcc}
\hline $\begin{array}{l}\text { Percentage of correct } \\
\text { answers }\end{array}$ & No. of questions & $\begin{array}{c}\text { Cumulative no. of } \\
\text { questions }\end{array}$ \\
\hline 100 & 8 & 8 \\
$90-99$ & 27 & 35 \\
$80-89$ & 16 & 51 \\
$70-79$ & 14 & 65 \\
$60-69$ & 6 & 71 \\
$50-59$ & 11 & 82 \\
$40-49$ & 9 & 91 \\
$30-39$ & 4 & 95 \\
$20-29$ & 4 & 99 \\
$10-19$ & 1 & 100 \\
$0-9$ & 0 & 100 \\
\hline
\end{tabular}


Table 3. Number of questions according to the correct answer rate in various domains

\begin{tabular}{lcc}
\hline Domain & No. of questions with a correct \\
answer rate of 50\% or less & NA & $\begin{array}{c}\text { No. of questions with a correct } \\
\text { answer rate of 90\% or more }\end{array}$ \\
\hline Role of the manuscript editor & 4 & 2 \\
General corrections & NA & 1 \\
Units and abbreviations & 2 & 5 \\
References & 1 & NA \\
Statistics & NA & 3 \\
Tables and figures & NA & 7 \\
Understanding the elements of a research article & 2 & 3 \\
Publication ethics and copyright & 2 & 5 \\
Technical factors related to publishing & 2 & 2 \\
Databases and citation indices & 5 & 1 \\
Korean grammar & 18 & 1 \\
English grammar and punctuation & 2 & 35 \\
Total & & 3 \\
\hline
\end{tabular}

NA, not available.

Four of the 12 questions from the units and abbreviations domain and 5 of the 15 from the English grammar and punctuation domain had a correct answer rate of under $50 \%$. Moreover, only 1 question from each domain had a correct answer rate of over $90 \%$. Therefore, these 2 domains are considered to be of the utmost importance and difficulty.

The domains of general corrections, references, understanding the elements of a research article, and publication ethics and copyright contained $9,8,8$, and 8 questions, respectively, all of which showed a correct answer rate of over $50 \%$. These 4 domains were low in difficulty, as more than $90 \%$ of the candidates chose the correct answers for 5, 5, 7, and 3 questions, respectively. All the candidates provided correct answers to questions such as the role of a ME, selecting the appropriate instructions for authors, data computation, and the use of punctuation marks in English. This occurred because the candidates had practical experience working in the journal publication field.

Based on this analysis, it is possible to identify priorities for the future education of KMEC holders. General corrections, references, understanding the elements of a research article, and publication ethics and copyright were the domains in which the candidates showed a high level of understanding. Meanwhile, units and abbreviations and English grammar and punctuation were the most troublesome areas for MEs who did not major in medicine or science. In particular, the use of punctuation in English contained the questions with the highest and lowest correct answer rates, signifying that
Korean editors had an uneven understanding of English punctuation.

\section{Limitations}

There are 3 major limitations to this examination. First, it was difficult to develop balanced questions due to the wide range of professional fields and skills of the candidates.

Second, this test was not focused on selecting a certain number of people through a relative evaluation. It was an absolute evaluation system aimed to evaluate the examinees' work ability and knowledge, resulting in relatively less effort invested in the difficulty control that would be needed to differentiate the levels of the candidates. Instead, the test makers focused more on making the test of moderate difficulty, as it was the first examination. At least 2 people were requested to produce questions on each topic to maintain balance.

Finally, an item analysis assessing the difficulty level of each item objectively and clearly was not conducted. Item analysis is the process of evaluating the reliability of an item. It is a significant aspect of ensuring the balance of an examination and ultimately increasing the degree of completion by identifying certain errors that may be present [3]. The KMEC is expected to become more objective by calculating an index of item difficulty, item discrimination, error attractiveness, and other parameters associated with classical test theory in the future. 


\section{Conclusion}

The KCSE implemented the KMEC system and developed an examination, aiming to establish a standard for MEs in Korea. The recent examination was very meaningful because it was the first test to lead to the qualification of MEs in the ME certification system that was introduced in Korea.

A KMEC holder is officially guaranteed to have the basic knowledge and skills required in a professional ME. The KCSE plans to hold the KMEC examination annually and to provide professional education and updated information regarding worldwide trends in manuscript editing.

The KCSE will expand the pool of exam questions by continually inventing new questions and revising existing ones. In order to achieve objectivity and reliability, a constant effort is required in the standardization and application of the KMEC system. A performance test of candidates' ability to search for various pieces of information and practical proofreading skills may also be included in the process in the future.

The establishment of this certification system is expected to strengthen individual capacities and to further develop science journal publication in Korea by expanding the field of manuscript editing.

\section{Conflict of Interest}

No potential conflict of interest relevant to this article was reported.

\section{Acknowledgments}

Jisoo Yoon, the manager of the Korean Council of Science Editors, provided the aggregate data on the results of the Korea Manuscript Editors Certification examination, and HyeMin Cho, a chair of the Committee on Manuscript Editing in the Korean Council of Science Editors, arranged the whole process and consulted on this article. The authors thank them deeply.

\section{References}

1. Korean Council of Science Editors. Domestic news. Implementation of the first Korea Manuscript Editors Certification examination [Internet]. Seoul: Korean Council of Science Editors; 2016 [cited 2017 Jan 22]. Available from: http://kcse.org/newsletter/SCBK1000193/

2. Yi HJ, Cho HM, Chung HK, Ahn HT, Kim MS, Seo YJ. Towards the implementation of a system for manuscript editor certification. Sci Ed 2016;3:3-12. http://doi.org/10.6087/ kcse. 56

3. Lim CW. Item analysis for selecting science gifted elementary school student. J Sci Educ 2010;34:155-63. http://doi. org/10.21796/jse.2010.34.1.155 